Analogisierung von binärer Codierung und Gesellschaftsstruktur bedürfen sicher weiterer Diskussion. Es wäre auch zu fragen, ob die theoretische Anlage seiner Analyse nicht konsequent dazu führen muss, Digitalität als Meta-Codierung moderner Gesellschaften zu begreifen - und damit doch mit einer zentralen Annahme der Gesellschaftstheorie Luhmanns zu brechen: der Annahme der Gleichrangigkeit der Systeme in der funktional differenzierten Gesellschaft. Auf diese Spur scheint auch das Argument zu führen, dass der ,Sinnüberschuss“ digitaler Technik auf der Gebrauchsebene des Internets eben jenseits dieses Mediums liegt (S. 264ff., 290, 315), und zwar in der Verwertung, Verwaltung und Steuerung der im Zuge des Gebrauchs produzierten Muster (von Interessen, Vorlieben, Bedürfnissen, Einstellungen und so weiter) insbesondere zu Vermarktungszwecken: vom Erleben zum Handeln (S. 249, 291). Aber das scheinen weitergehende Fragen.

Nassehis „Muster“ besticht nicht nur durch mustergültige Lesbarkeit, sondern durch die Originalität seiner leitenden Fragestellung. Der Band eröffnet nichts weniger als die Pforten zu einer differenzierungsanalytisch angelegten Soziologisierung des Nachdenkens über die seit einiger Zeit unter dem Label „Digitalisierung“ zumeist kulturpessimistisch beschriebenen Prozesse: Ein sehr lesenswertes Buch zur digitalen Genealogie der modernen Gesellschaft.

\title{
Heyer, Andreas: Die Verfassung der Jakobiner von 1793 und ihr historischer Kontext. (Staatsverständnisse, Bd. 131), 313 S., Nomos, Baden-Baden 2019.
}

\section{Hans-Ulrich Thamer}

Angenommen: 17. Dezember 2020 / Online publiziert: 9. Februar 2021

(C) Der/die Autor(en) 2021

Mit der französischen Verfassung von 1793 verbindet sich eine widersprüchliche Geschichte. Sie entstand inmitten der heftigsten inneren Machtkämpfe zwischen den Fraktionen des revolutionären Konvents und wurde am 24. Juni, nach der Machtergreifung der Jakobiner, zwar beschlossen, aber nie wirklich in Kraft gesetzt. Der nun jakobinisch beherrschte Konvent setzte sie vielmehr in den Machtkämpfen mit den Girondisten als wirksame Waffe zur Legitimitätsbeschaffung ein.

H.-U. Thamer $(\square)$

Westfälische Wilhelms-Universität Münster, Münster, Deutschland

E-Mail: thamer@uni-muenster.de 
$\mathrm{Zu}$ den Widersprüchen gehört, dass die suspendierte Verfassung nach dem Sturz von Maximilien de Robespierre zum Bezugspunkt der antithermidorianischen Opposition wurde, die „Brot und die Verfassung von 1793“ forderte. Zugleich blieb die jakobinische Verfassung bis weit in das 19. Jahrhundert Modell für eine demokratische, republikanische Verfassung. Wenngleich sie in ihrer Entstehung viele Züge eines Kompromisses trug, stellte sie auch nach dem Urteil der modernen Verfassungsgeschichte eine bemerkenswerte Leistung dar. Als erste Verfassung überhaupt gründete sie sich allein auf die ,Vernunft“ und nicht mehr auf Gott. Sie beanspruchte „im Namen des Volkes“ geschaffen zu sein. Durch ihren Artikel IV erhielt zudem ,jeder in Frankreich geborene und wohnhafte Mann“ ab 21 Jahren und jeder Ausländer, der mehr als ein Jahr in Frankreich lebte und arbeitete, das volle Bürgerund Wahlrecht. Artikel IX der neuen Déclaration des droits versprach, dass ,das Recht die allgemeine und die individuelle Freiheit vor der Unterdrückung durch die Regierenden schützen soll“".

Die Verfassung von 1793 verdeutlicht überdies, wie tief inzwischen die Kluft zwischen Revolution und Verfassung geworden war. Alle Bemühungen, die revolutionäre Bewegung in eine Verfassungsordnung einzubinden, scheiterten. Um ihren historischen Ort bestimmen zu können, muss ihre Entstehung und Bedeutung in ihren historischen Kontext eingeordnet werden. Das ist auch die methodische Prämisse der Studie von Andreas Heyer, der zunächst den ,geschichtlichen Hintergrund“ sowie die „Verknüpfung der politischen, ideologischen und gesellschaftlichen Bereiche“ (S. 42) beschreiben will, ,vor denen sich die Verfassungsdebatte abspielte“ (S. 10). Das ist sicherlich verdienstvoll, denn nach Heyers Urteil verzichten bisherige wissenschaftliche Kommentierungen der jakobinischen Verfassung auf eine solche Kontextualisierung. Wenn er allerdings der jüngsten kommentierten Edition des Verfassungsentwurfs der Gironde vom Februar 1793, herausgegeben von Andreas Kley und Richard Amstutz (St. Gallen 2011), im selben Atemzug vorwirft, die „Lektüre der Forschungsliteratur [sei] nicht ihre Sache“ (S. 150), dann fällt dieses harsche Urteil auf ihn selbst zurück. Denn für ihn zählen bei seiner historischen Einordnung der Verfassungsdebatte neben den ,großen Historikern“ des 19. Jahrhunderts - gemeint sind vor allem François-Alphonse Aulard, Jules Michelet, Octave Aubry und François-Auguste Mignet - nur die sozialistischen Revolutionshistoriker des 20. Jahrhunderts von Albert Mathiez bis Albert Soboul. Die durch politik- und sozialgeschichtliche Studien hervorgetretenen und die aktuelle Forschungsdiskussion prägenden französischen und amerikanischen, gerne als ,,revisionistisch“ charakterisierten Historiker von François Furet bis Jonathan Israel tauchen (mit der Ausnahme von Keith Baker und seinen Condorcet-Studien) so gut wie gar nicht in der Literaturliste und noch weniger in der Interpretation von Heyer auf. An deutschsprachiger Forschungsliteratur kennt er nur die - sicherlich verdienstvolle - marxistische DDRForschung, alle anderen Darstellungen hält er offensichtlich für irrelevant.

Diese teilweise einseitige Literaturauswahl, die an die Zeiten des Neomarxismus der 1960er und 1970er Jahre erinnert, findet ihren Niederschlag nicht nur in der einleitenden kurzgefassten Abhandlung der Revolutionsgeschichte von 1789 bis 1792/93, sondern auch in Heyers detaillierter Darstellung der kontroversen Verfassungsdebatte von 1793, in der er mit guten Gründen der Diskussion von Condorcets politischer Philosophie und seines Verfassungsentwurfs wie der politischen Philo- 
sophie und verfassungspolitischen Strategie seines Gegenspielers Robespierre die entscheidende Rolle zuweist. Weiterführend sind zudem die Kapitel über alternative linke Verfassungskonzepte von Jacques Roux und François-Joseph L'Ange und die definitive, vor dem Hintergrund der dramatischen Krise der Revolution zu einem Kompromiss führende, Verfassungsdebatte bis hin zur Suspendierung des durch einen Volksentscheid legitimierten Verfassungstextes. Zuzustimmen ist auch Heyers Urteil, dass es sich bei der Verfassung von 1793, die auf einen heftig umkämpften Entwurf von Condorcet zurückzuführen ist, dank ihrer ,innovativen Elemente“ um einen ,großen Wurf“ (S. 143) handelt.

Wenn der Autor dann allerdings Condorcets Ziel, mit der Verfassung die Revolution zu beenden (was das Ziel fast aller Verfassungsentwürfe war) und die sansculottische soziale Revolution zu verhindern, mit dem Vorwurf der „Klassenpolitik“ kommentiert und sich in dem apodiktischen Satz gefällt: „Klassenpolitik bleibt Klassenpolitik, egal welches Gewand der Redner trägt“, dann lässt er sich vom ,marxistischen Dogma“ seiner von ihm favorisierten Autoren verleiten, ohne die längst vorliegenden sozialhistorischen Befunde über die soziale Rekrutierung der Girondisten und ebenso wenig der Montagnards zu beachten. Doch lässt sich die ,neo-jakobinische“ (Furet) These von der Determinierung des Politischen durch das Soziale in dieser Einfachheit nicht aufrechterhalten, wie auch Heyers Diktum, der Krieg gehöre zu den ,kleinen Freuden der Bourgeoisie“ (S. 68) weniger von historischer Differenzierung als von dogmatischem Denken bestimmt ist. Ein ökonomischer Determinismus, wie ihn Heyer vertritt, würde die Rolle von Mentalitäten und politischen Haltungen, die schwankend und situationsabhängig sein können, leugnen. Allerdings zeigt gerade die Politik der Montagnards solche Schwankungen und Anpassungen, die sich nicht allein mit ihrem vermeintlichen „Klasseninteresse“ erklären lassen. Robespierres Macht lag vielmehr in seiner politischen Fähigkeit, die Dynamik der Revolution zu begreifen und sie für sich zu nutzen. Trotz seiner eigenen bürgerlichen Herkunft und der seiner Gefolgsleute konnte er sich darum, im Gegensatz zu den ebenfalls bürgerlichen Girondisten, erfolgreich zum Sprecher der sozialen Interessen ,des Volkes‘ machen.

Funding Open Access funding enabled and organized by Projekt DEAL.

Open Access Dieser Artikel wird unter der Creative Commons Namensnennung 4.0 International Lizenz veröffentlicht, welche die Nutzung, Vervielfältigung, Bearbeitung, Verbreitung und Wiedergabe in jeglichem Medium und Format erlaubt, sofern Sie den/die ursprünglichen Autor(en) und die Quelle ordnungsgemäß nennen, einen Link zur Creative Commons Lizenz beifügen und angeben, ob Änderungen vorgenommen wurden.

Die in diesem Artikel enthaltenen Bilder und sonstiges Drittmaterial unterliegen ebenfalls der genannten Creative Commons Lizenz, sofern sich aus der Abbildungslegende nichts anderes ergibt. Sofern das betreffende Material nicht unter der genannten Creative Commons Lizenz steht und die betreffende Handlung nicht nach gesetzlichen Vorschriften erlaubt ist, ist für die oben aufgeführten Weiterverwendungen des Materials die Einwilligung des jeweiligen Rechteinhabers einzuholen.

Weitere Details zur Lizenz entnehmen Sie bitte der Lizenzinformation auf http://creativecommons.org/ licenses/by/4.0/deed.de. 